\title{
Effects of Autapse and Channel Blockage on Firing Regularity in a Biological Neuronal Network
}

\author{
Rukiye UZUN ${ }^{1}$, Mahmut OZER ${ }^{1}$
}

\author{
${ }^{1}$ Bulent Ecevit University, Zonguldak, Turkey \\ rukiye.uzun@beun.edu.tr, mahmutozer2002@yahoo.com
}

\begin{abstract}
In this paper; the effects of autapse (a kind of self-synapse formed between the axon of the soma of a neuron and its own dendrites) and ion channel blockage on the firing regularity of a biological small-world neuronal network, consists of stochastic Hodgkin-Huxley neurons, are studied. In this study, it is assumed that all of the neurons on the network have a chemical autapse and a constant membrane area. Obtained results indicate that there are different effects of channel blockage and parameters of the autapse on the regularity of the network, thus on the temporal coherence of the network. It is found that the firing regularity of the network is decreased with the sodium channel blockage while increased with potassium channel blockage. Besides, it is determined that regularity of the network augments with the conductance of the autapse.
\end{abstract}

Keywords: Autapse, channel blocking, ion channel noise, small-world neuronal netwoks.

\section{Introduction}

The nervous system, having a complex biologic structure, comprises of billions of nerve cells (neurons) and synaptic connections between them [1]. In this complex structure, it is believed that the signal communication (i.e. information transmission) can be carried out by synaptic coupling [2, 3]. Synapses are essentially categorized in two different types: electrical and chemical synapses [4]. However, several decades ago, neurobiologists found out that some neurons could be connected to itself which forms a time-delayed feedback mechanism on a cellular level [5-9]. These self-synapses named as an autapse and were proposed by Van der Loss and Glaser in 1972 [10]. Although autapses have an unusual (odd) structure, they have been observed commonly in various brain areas, such as neocortex, hippocampus and cerebellum etc. [4, 11-13, Wang and Chen 2015, Wang et al 2016].

Besides, it has been experimentally presented that these type of synapses have significant effects on the firing dynamics of neurons. For example, Bacci and Huguenard (2006) showed that the autaptic connections have a vital role on determining the precise spike timing of inhibitory interneurons in neocortex [14]. Rusin et al. [15] demonstrated that usage of continuous timedelayed feedback stimulation can arrange the synchronization of spikes in cultured neurons. In addition to these experimental studies, there is also numerous studies that investigate the impacts of autapse on firing dynamics of the neuron with computational neuronal models [16-25]. In this context, Li et al [16] investigated the effect of ion channel noise on dynamics of neuron in the presence of autapse based on a stochastic Hodgkin-Huxley (HH) model. They found that autapse is reduced the spontaneous spiking activity of neuron at characteristic frequencies by inducing bursting firing and multimodal interspike interval distribution. Connelly [17] revealed that autapses augment the gamma oscillations of interneurons, known as basket cells, in a Wang-Buzsáki model during the gamma oscillations. Wang et al [18] discovered that autapses causes to switch the dynamics of electrical activity of Hindmarsh-Rose model among different firing patterns (quiescent, periodic and chaotic). In another study, it is demonstrated that autapses can regulate the modelocking behaviors of a $\mathrm{HH}$ model subjected to sinusoidal stimulus, especially by autaptic delay time [19]. Recently, Yilmaz and Ozer [20] consider the weak signal detection performance of a stochastic $\mathrm{HH}$ model with regard to an electrical autapse. They obtained that the detection performance can be healed or deteriorated depending on autapses' parameters. Similar studies concerning weak signal detection performance have been realized on smallworld (SW) and scale-free (SF) neural networks by Yilmaz et al and have been obtained analogous results [21, 22]. Wang and Gong [23] have shown that both multiple coherence resonance (MCR) and synchronization transitions are occurred because of autpases, in SW neuronal network. Recently, Wang and Chen revealed that autapses provide an opportunity to engineer the response of a $\mathrm{HH}$ model to subthreshold stimulus [24]. Moreover, in this study, it is revealed out that the detection of subthreshold stimulus is increased due to autapse. More recently, the impacts of both electrical and chemical autapses on the temporal coherence 
of a single $\mathrm{HH}$ model and SF neuronal network is analyzed by Yilmaz et al [25]. They obtained that the dynamics of neuron or network change prominently with the proper choice of autaptic parameters.

Nonetheless, it is well known that neuronalinformation processing operates under diverse noise sources, which has therapeutic influences contrary to expectations [26]. The most important noise source in neural systems is originated in voltage-gated ion channels due to their random transitions between conducting and non-conducting states [27]. The strength of channel noise is related with the numbers of ion channels on membrane, thus membrane area. But, its real effect on neuron's dynamics is determined by the number of active ion channels participating in the generation of spikes [28]. Therefore, addressing impacts of the number of active ion channels is important especially to uncover the role of specific ion channel noise on neuronal spiking activity. Experimental studies indicate that some neurotoxins such as tetraetylammonium (TEA) ve tetratoxin (TTX) can alter the properties of ion channels [29]. For a given membrane, by a fine-tuned addition of these toxins a certain portion of potassium- and sodium ion channels could be disabled or blocked and hence the number of active (working) ion channels can be reduced. There are also plenty of studies based on different computational neuron models, where the impacts of changing the number of particular ion channels on firing dynamics of a single neuron or neuronal networks' is examined [3038]. In these studies, it is uncovered that channel blocking has crucial impacts on firing dynamics.

In this study, different from the above studies, we investigate how the firing dynamics of Newman-Watts SW neuronal network, consisting of stochastic $\mathrm{HH}$ neurons, change in the presence of both ion channel blocking and autapse. For this aim, we assume that all neurons on the network have a chemical autapse and are exposed to same ratio of ion channel blocking.

\section{Model and Method}

Time evolution of membrane potentials of NewmanWatss network of $\mathrm{HH}$ neurons in the presence of chemical autapse is given as follows [16, 21-23, 25]:

$C_{m} \frac{d V_{i}}{d t}=-G_{N a}\left(m_{i}, h_{i}\right)\left(V_{i}-V_{N a}\right)-G_{K}\left(n_{i}\right)\left(V_{i}-\right.$
$\left.V_{K}\right)-G_{L}\left(V_{i}-V_{L}\right)+\sum \varepsilon_{i j}\left(V_{j}(t)-V_{i}(t)\right)+I_{a u}$

$\left.V_{K}\right)-G_{L}\left(V_{i}-V_{L}\right)+\sum \varepsilon_{i j}\left(V_{j}(t)-V_{i}(t)\right)+I_{a u t_{i}}$

Here $C_{m}$ is the membrane capacitance per unit area, $V_{i}$ denotes the membrane potential of neuron $i=1, \ldots, N(\mathrm{~N}$ shows the network size) in $\mathrm{mV}$, and $V_{N a}=50 \mathrm{mV}, V_{K}=-$ $77 \mathrm{mV}$ and $V_{L}=54.4 \mathrm{mV}$ are the equilibrium potential of sodium, potassium and leakage ion channels, respectively. $\varepsilon_{i j}$ denotes the coupling strength between neurons $\mathrm{i}$ and $\mathrm{j}$, whereby we set $\varepsilon_{i j}=0.1$ if there is a connection between neurons or $\varepsilon_{i j}=0$ otherwise.

$I_{\text {auti }}$ is the delayed feedback current related to the autaptic connection of neuron $i$. The autaptic current of a simplified neuron model can be described by two different forms. One is linear coupling (i.e. electrical) and the other is nonlinear coupling (i.e. chemical). In this study we use chemical autaptic current which is given by the following two equations $[21,23,41]$ :

$I_{\text {aut }_{i}}=-g_{\text {aut }}\left[V_{i}(t)-V_{\text {syn }}\right] S(t-\tau)$
$S_{i}(t-\tau)=1 /\left\{1+\exp \left[-k\left(V_{i}(t-\tau)-\theta\right]\right\}\right.$

Here $g_{\text {aut }}$ is the autaptic intensity and $\tau$ is the delay time which is taken as $10 \mathrm{~ms}$ throughout the study. $V_{\text {syn }}$ represents the autaptic reversal potential and its value varies depending on whether the autapse is excitatory $\left(V_{s y n}=2 \mathrm{mV}\right)$ or inhibitory $\left(V_{s y n}=-2 \mathrm{mV}\right)$. We assumed that all neurons have excitatory chemical autapse with the same autaptic parameters. The other parameters' values, in equation (2), is set as $k=8, \theta=-0.25$. More interpretations of all parameters in Eq. 2 can be found at Ref. [41].

In equation (1), $G_{N a}, G_{K}$ and $G_{L}$ denote sodium, potassium and leakage ion channel conductance, respectively. In the model, the leakage conductance is constant, $\mathrm{G}_{\mathrm{L}}=0.3 \mathrm{mScm}^{-2}$, whereas the others dynamically change as follows $[30,34,37,38]$ :

$G_{N a}\left(m_{i}, h_{i}\right)=g_{N a}^{\max } \chi_{N a} m_{i}^{3} h_{i}, G_{K}\left(n_{i}\right)=g_{K}^{\max } \chi_{K} n_{i}^{4}$

where $g_{N a}{ }^{\max }=120 \mathrm{mScm}^{-2}$ and $g_{K}{ }^{\max }=36 \mathrm{mScm}^{-2}$ is the maximal conductance of the corresponding ion channel. $\chi_{i}$ $i=(N a, K)$ is a scaling factor, which equals to the ratio of active (non-blocked) $i$-type ion channel numbers to total $i$ type ion channel number within a given membrane size [30, 31]. This factor is confined to unit interval and $i$-type ion channel blocking level gets higher as the factor approaches zero. In equation ( 3$) ; n_{i}, m_{i}$ and $h_{i}$ are dimensionless variables that represent for the potassium ion channel activation, sodium ion channel activation and inactivation, respectively. The dynamics of these variables is described by the following Langevin equation for a finite membrane size [42]:

$$
\begin{aligned}
\frac{d x_{i}}{d t} & =\alpha_{x_{i}}(V)\left(1-x_{i}\right)-\beta_{x_{i}}(V) x_{i}+\xi_{x_{i}}(t), \\
x_{i} & =m, n, h
\end{aligned}
$$

Here $\alpha_{x i}$ and $\beta_{x i}$ are the voltage-dependent opening and closing rates of $x_{i}$. $\zeta_{x i}(t)$ is the zero mean Gaussian white noises whose auto-correlation functions given as follows [42]:

$$
\begin{aligned}
& \left\langle\xi_{x}(t) \xi_{x}\left(t^{\prime}\right)\right\rangle=\frac{2 \alpha_{x} \beta_{x}}{N_{j} \chi_{j}\left(\alpha_{x}+\beta_{x}\right)} \delta\left(t-t^{\prime}\right) \\
& N_{j}=\rho_{j} S ; j=N a, K ; x=m, n, h
\end{aligned}
$$

where $S$ denotes the membrane size, $N_{j}$ and $\rho_{j}$ are the total number and density of corresponding ion channel, respectively [30, 31]. Let $\rho_{N a}=60 \mu m^{-2}$ and $\rho_{K}=18 \mu m^{-2}$. In the model, it is assumed that sodium and potassium ion channel densities are homogenous.

The considered neuronal network is Newman-Watts (NW) small world network [39]. The network is constituted as follows. It starts with a regular ring consisting of $N=60$ identical $\mathrm{HH}$ neurons in which each neuron has connections with its $k=2$ nearest neighbors. Then new links are randomly 
added between non- adjacent neighbors. The randomness expressed with the fraction of shortcuts corresponding to $p=M /(N(N-1))$ ( $M$ is the number of new links). The constructed network exhibits smallworld properties when $\mathrm{p}$ is confined to unit interval. In this study, we construct the network with $p=0.15$.

In order to analyze the impacts of ion channel blockage and autapses on dynamics of network, we calculate the collective firing regularity of the network. To do so, we first obtain the firing times of neurons on the network via the average membrane potential () and then calculate the spontaneous collective spiking regularity with:

$\lambda=\frac{\langle I S I\rangle}{\sqrt{\left\langle\left(I S I^{2}\right)\right\rangle-\langle I S I\rangle^{2}}}$

Here $\langle I S I\rangle$ and $\left\langle I S I^{2}\right\rangle$ are the mean and meansquared interspike intervals, respectively. The firing pattern of network gets more regular, as $\lambda$ increases. In the following results, spike times are determined by $V_{\text {avg }}(t)$ crosses the detection threshold 0 from below and also each $\lambda$ value is calculated by averaging over 20 different network realization.

\section{Results and Discussion}

In a recent study, Wang and Gong [23] have investigated how the temporal coherence (namely, firing regularity) and synchronization of Newman-Watts SW neural network alter in the presence of autaptic selfdelayed feedback. In the light of their study, here, we systemically investigate the effects of potassium and sodium ion channel blocking on the collective firing regularity of network $(\lambda)$ as a function of autaptic intensity $\left(g_{\text {aut }}\right)$ at a constant autaptic time delay $(\tau=$ $10 \mathrm{~ms}$ ). To show effects of each specific ion channels, the density of one channel type is varied while keeping the other channel type equals one $\left(\chi_{K}=1\right.$ or $\left.\chi_{N a}=1\right)$. Besides, throughout this study, we set $S=6 \mu \mathrm{m}^{2}$ because of the optimum temporal coherence is obtained $[32,33]$. Then, we calculate $\lambda$ with respect to $g_{\text {aut }}$ for various $\chi_{K}$ and $\chi_{N a}$.

We first investigate how $\lambda$ changes with the nonblocked potassium ion channel fraction (i.e. active potassium ion channel, $\chi_{\mathrm{K}}$ ) as a function of $g_{a u t}$, as shown in Figure 1. From the obtained results, one can see that the reduction of the potassium ion channels has two essential effect on $\lambda$. First is that $\lambda$ increases with the increment of the potassium ion channel blocking (i.e. decrease in $\chi_{K}$ ) for a constant $g_{a u t}$. Later, for a constant $\chi_{\mathrm{K}}, \lambda$ displays an exponential raise beyond a certain $g_{\text {aut }}$. This value of $g_{\text {aut }}$ moves to smaller values as $\chi_{K}$ decreases.

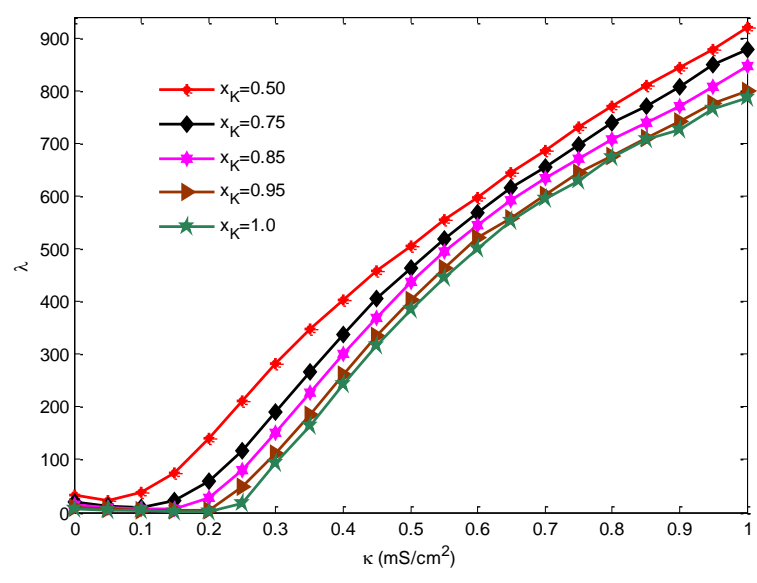

Figure 1. Dependence of collective firing regularity $\lambda$ on autaptic intensity $g_{\text {aut }}$ for various potassium channel block ratios $\chi_{\mathrm{K}}$ at a constant autaptic time delay $\tau=10 \mathrm{~ms}$. $\left(\chi_{N a}=1.0, p=0.15, \varepsilon=\right.$ $\left.0.1, S=6 \mu m^{2}\right)$.

To gain more insight into the dependence of collective firing regularity on $\chi_{K}$, we further analyze the variation of $\lambda$ as a function of $\chi_{\mathrm{K}}$. We set autaptic parameters as $\tau=10 \mathrm{~ms}$ and $g_{a u t}=0.15 \mathrm{mScm}^{-2}$. Obtained result is presented in Figure 2. It can be obviously seen that $\lambda$ increases as $\chi_{K}$ decreases. The potassium ion channel blocking causes the increment at the excitability of neurons in the network, and because of this, collective firing regularity of network becomes more regular. Besides, the presence of autaptic connections in the network and the increment of autaptic intensity $g_{\text {aut }}$ augments the collective firing dynamics of the network. Briefly, one can conclude that the presence of both potassium ion channel blocking and autaptic connections have therapeutic effects on the temporal coherence of network.

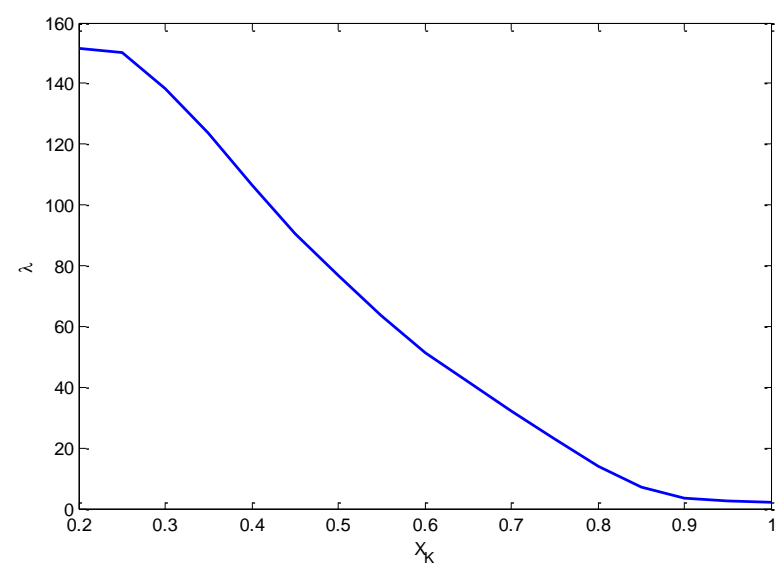

Figure 2. Dependence of collective firing regularity $\lambda$ on potassium channel block ratios $\chi_{K}$, when the autaptic parameters are constant $\left(\tau=10 \mathrm{~ms}, g_{\text {aut }}=0.15 \mathrm{mScm}^{-2}\right) . \quad\left(\chi_{\mathrm{Na}}=1.0, p=0.15, \varepsilon=\right.$ $\left.0.1, S=6 \mu m^{2}\right)$.

After determining the impacts of potassium ion channels on temporal coherence of network in the presence of autapse, we examine the effects of sodium ion channels known as another important ion channels at determining the firing dynamics of neuron. For this, we implement similar simulations for sodium ion channels as we did in case of 
potassium channel block. In the simulations we consider $\chi_{\mathrm{Na}}>0.7$, because the average membrane potential $V_{\text {avg }}$ do not include spikes under below this $\chi_{\mathrm{Na}}$ value. In Figure 3, it can be clearly seen that reduction in active sodium ion channels influences the temporal coherence of network $(\lambda)$ oppositely compared to the case of potassium ion channel blockage. Namely, decreasing the $\chi_{\mathrm{Na}}$ disrupts the $\lambda$. But this destructive influence vanishes at smaller $g_{\text {aut }}$ and besides, the value of $g_{\text {aut }}$ where the sodium ion channel blockage does not make important variation on $\lambda$ increases as $\chi_{\mathrm{Na}}$ decreases. As a result, the sodium ion channel block degrades the temporal coherence of the network in the presence of autaptic connections.

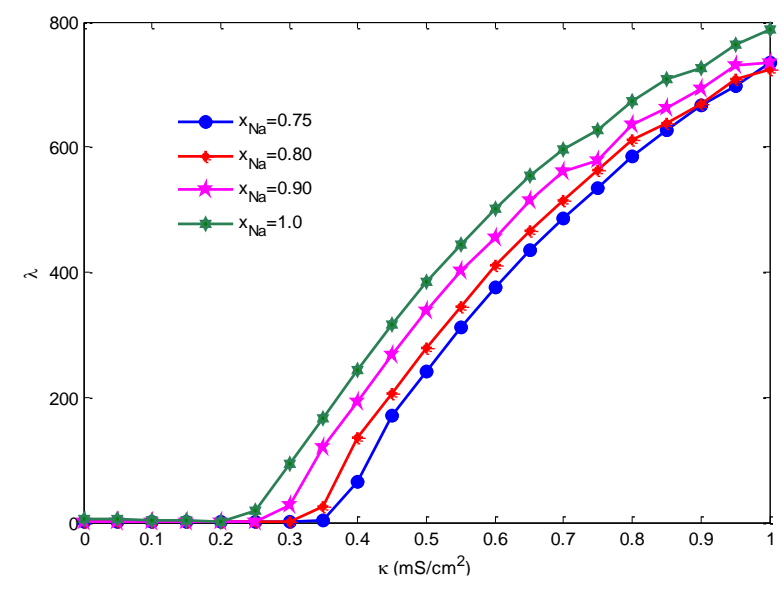

Figure 3. Dependence of collective firing regularity $\lambda$ on autaptic intensity $g_{\text {aut }}$ for various sodium channel block ratios $\chi_{N a}$ at a constant autaptic time delay $\tau=10 \mathrm{~ms}$. $\left(\chi_{K}=1.0\right.$, $p=0.15, \varepsilon=0.1, S=6 \mu m^{2}$ ).

In order to show prominently the impact of sodium ion channel blockage on temporal coherence of the network, we also give the simulation results how $\lambda$ changes as a function of $\chi_{N a}$ at constant autapse parameters $(\tau=$ $10 \mathrm{~ms}, g_{\text {aut }}=0.5 \mathrm{mScm}^{-2}$ ) (Figure 4). From Figure 4 , one can see clearly the destructive impact of the reduction of active sodium ion channel.

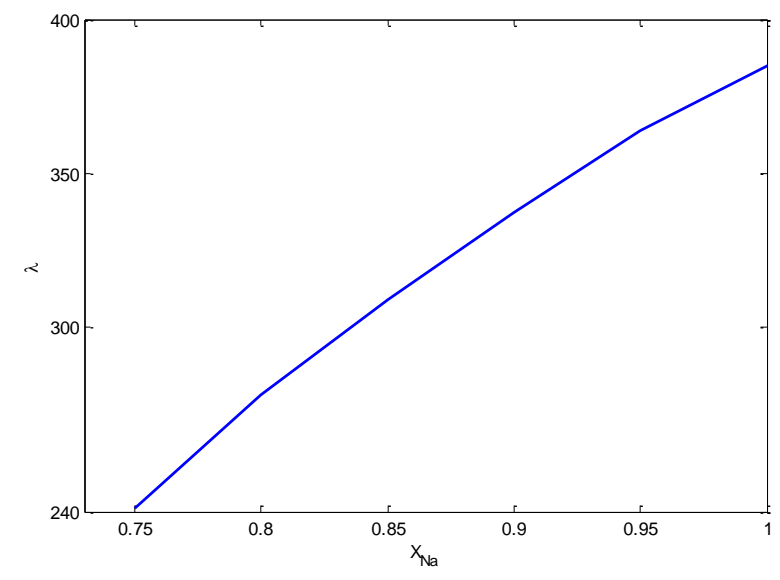

Figure 4. Dependence of collective firing regularity $\lambda$ on sodium channel block ratios $\chi_{\mathrm{Na}}$ when the autaptic parameters are constant $\left(\tau=10 \mathrm{~ms}, g_{a u t}=0.5 \mathrm{mScm}^{-2}\right) .\left(\chi_{\mathrm{K}}=1.0\right.$, $\left.p=0.15, \varepsilon=0.1, S=6 \mu m^{2}\right)$.

\section{Conclusions}

In sum, we investigate the collective firing regularity of NW small-world neuronal networks in the presence of both autapse and ion channel block. Comparing the effect of potassium and of sodium channel block, one can see that they have much different influences on temporal coherence of the network. For potassium ion channel block, firing regularity increases, while for sodium channel block, it affects negatively. This could be because of the discriminating impacts of potassium and sodium ion channel blockage on the firing dynamics $[30,31]$.

\section{References}

[1] R. Uzun, "Biyolojik Nöral Ağlarda Latans Dinamiklerinin Analizi", PhD thesis, Elec Depar. of EEE, BEU, Zonguldak., Turkey, 2014.

[2] E. R. Kandel, J. H. Schwartz, T. M. Jessel, S. A. Siegelbaum, and A. J. Hudspeth, "Principles of Neural Science 4th Edition ", McGraw-Hill Medical, New York, England, 2000.

[3] H. Qin, J. Ma, C. Wang, and Y. Wu, "Autapse-induced sprial wave in network of neurons under noise", PlosOne, vol. 9, no. 6, pp. 1-9, 2014.

[4] E. P. Furshpan, and D. D. Potter, "Transmission at the giant motor synapses of the crayfish", J. Physiol., vol.145, pp. 289345, 1959.

[5] A. B. Karabelas, and D. P. Purpura, "Evidence for autapse in the substantianigra", Brain Res., vol. 200, no. 2, pp. 467-473, 1980.

[6] G. Támas, E. H. Buhl, and P. Somogyi, "Massive autaptic selfinnervation of GABAergic neurons in cat visual cortex", J. Neurosci., vol. 17, no. 16, pp. 6352-64, 1997.

[7] J. M. Bekkers, "Neurophysiology: are autapses prodigal synapses?", Curr. Biol., vol. 8, no. 2, pp. R52-55, 1998.

[8] J. M. Bekkers, "Synaptic transmission: functional autapses in the cortex", Curr. Biol., vol. 13, no. 11, pp. R433-435, 2003.

[9] K. Ikeda, and J. M. Bekkers, "Autapses", Curr. Biol., vol. 16, no. 9, pp. R308, 2006.

[10] H. Van der Loos, and E. M. Glaser, "Autapses in neocortex cerebri: synapses between a pyramidal cell's axon and its own dendrites", Brain Res., vol. 48, pp. 355-360, 1972.

[11] J. Lübke, H. Markram, M. Frotscher, and B. Sakmann, "Frequency and dendritic distribution of autapses established by layer 5 pyramidal neurons in the developing rat neocortex: Comparison with synaptic innervation of adjacent neurons of the same class", J. Neurosci., vol. 16, pp. 3209-3218, 1996.

[12] M. H. Flight, "Neuromodulation: Exerting self-control for persistence", Nat. Rev. Neurosci., vol. 10, pp. 316, 2009.

[13] T. Branco, and K. Staras, "The probability of neurotransmitter release: Variability and feedback control at single synapses", Nat. Rev. Neurosci., vol. 10, pp. 373-383, 2009.

[14] A. Bacci, and J. R. Huguenard, "Enhancement of spike-timing precision by autaptic transmission in neocortical inhibitory interneurons", Neuron, vol. 49, pp. 119-130, 2006.

[15] C. G. Rusin, S. E. Johnson, J. Kapur, and J. L. Hudson, "Engineering the synchronization of neuron action potentials using global time-delayed feedback stimulation", Phys. Rev. E, vol. 84, pp. 066202, 2011.

[16] Y. Li, G. Schmid, P. Hänggi, and L. Schimansky-Geier, "Spontaneous spiking in an autaptic Hodgkin-Huxley setup", Phys. Rev. E, vol. 82, pp. 061907, 2010.

[17] W. M. Connelly, "Autaptic connections and synaptic depression constrain and promote gamma oscillations", PlosOne, vol. 9, pp. e89995, 2014. 
[18] H. Wang, J. Ma, Y. Chen, and Y. Chen, "Effect of an autapse on the firing pattern transition in a bursting neuron", Commun. Nonlinear Sci. Numer. Simul., vol. 19, pp. 3242-3254, 2014.

[19] H. Wang, Y. Sun, Y. Li, and Y. Chen, "Influence of autaptic self-feedback on mode-locking structure of a Hodgkin-Huxley neuron under sinusoidal stimulus", J. Theoret. Biol., vol. 358, pp. 25-30, 2014.

[20] E. Yilmaz, and M. Ozer, "Delayed feedback and detection of weak periodic signals in a stochastic Hodgkin-Huxley neuron", Physica A, vol. 421, pp. 455-462, 2015.

[21] E. Yilmaz, V. Baysal, M. Ozer, and M. Perc, "Autaptic pacemaker mediated propagation of weak rhythmic activity across small-world neuronal networks", Physica A, vol. 444, pp. 538-546, 2016.

[22] E. Yilmaz, V. Baysal, M. Perc, and M. Ozer, "Enhancement of pacemaker induced stochastic resonance by an autapse in a scale-free neuronal networks", Sci. China Technol. Sc., vol. 59, no. 3, pp. 364-370, 2016.

[23] Q. Wang, and Y. Gong, "Multiple coherence resonance and sychronization transitions induced by autaptic delay in Newman-Watts neuron networks", Appl. Math. Model., vol. 40, pp. 7147-7155, 2016.

[24] H. Wang, and Y. Chen, "Response of autaptic HodgkinHuxley neuron with noise to subthreshold sinusoidal signals", Physica A, vol. 462, pp. 321-329, 2016.

[25] E. Yilmaz, M. Ozer, V. Baysal, M. Perc, and M. Perc, "Autapse-induced multiple coherence resonance in single neurons and neuronal networks", Sci. Rep., vol. 6, pp. 30914, 2016.

[26] P. Hänggi, "Stochastic resonance in biology", Chem. Phys. Chem., vol. 3, pp. 285-290, 2002.

[27] P. N. Steinmetz, A. Manwani, C. Koch, M. London, and I. Segev, "Subthreshold voltage noise due to channel fluctuations in active neuronal membranes", J. Comput. Neurosci., vol. 9, pp. 133-148, 2000.

[28] E. Schneidman, B. Freedman, and I. Segev, "Ion channel stochasticity may be critical in determining the reliability and precision of spike timing", Neural Comput., vol. 10, pp. 1679-1694, 1998.

[29] B. Hille, "Ionic Channels of Excitable Membranes", Unv. of Washington, Sinauer Press, USA, 1992.

[30] G. Schmid, I. Goychuk, and P. Hänggi, "Effect of channel block on the spiking activity of excitable membranes in a stochastic Hodgkin-Huxley model", Phys. Biol., vol. 1, no. 1-2, pp. 61-66, 2004.

[31] G. Schmid, I. Goychuk, and P. Hänggi, "Controlling the spiking activity in excitable membranes via poisoning", Physica A, vol. 344, no. 3-4, pp. 665-670, 2004.

[32] Y. B. Gong, B. Xu, X. G. Ma, and J. Q. Han, "Effect of channel block on the collective spiking activity of coupled stochastic Hodgkin-Huxley neurons", Sci. China Ser. B, vol. 51, no. 4, pp. 341-346, 2008.

[33] M. Ozer, M. Perc, and M. Uzuntarla, "Controlling the spontaneous spiking regularity via channel blocking on Newman-Watts networks of Hodgkin-Huxley neurons", Europhys. Lett., vol. 86, no. 4, pp. 40008-14, 2008.

[34] M. Uzuntarla, R. Uzun, E. Yilmaz, M. Ozer, and M. Perc, "Noise-delayed decay in the response of a scale-free neuronal network", Chaos Soliton Fract., vol. 56, pp. 202208, 2013.

[35] J. Ma, H. Long, C. N. Wang, and Z. S. Pu, "Robustness, death of spiral wave in the network of neurons under partial ion channel block", Commun. Theor. Phys., vol. 59, no. 2, pp. 233-242, 2013.

[36] L. Huang, J. Ma, J. Tang, and F. Li, "Transition of ordered waves in neuronal network induced by diffusive poisoning of ion channels", J. Biol. Syst., vol. 21, no. 01, pp. $1350002,2013$.

[37] R. Uzun, M. Ozer, and M. Perc, "Can scale-freeness offset delayed signal detection in neuronal networks?", Eur. Phys. J. A, vol. 105, no. 6, pp. 60002, 2014.

[38] R. Uzun, and M. Ozer, "The effect of channel blocking on first spike timing", in IEEE 23nd Signal Processing and Communications Applications Conference, Malatya, Turkey, 2015, pp. 1191-1199.

[39] M. E. J. Newman, and D. J. Watts, "Scaling and percolation in the small-world network model", Phys. Rev. E, vol. 60, no. 1, pp. 7332-7342, 1999.

[40] A. Hodgkin, and A. Huxley, "A quantitative description of membrane current and its application to conduction and excitation in nerve", J. Physiol., vol. 117, no. 4, pp. 500-544, 1952.

[41] N. Burić, K. Todorović, and N. Vasović, "Synchronization of bursting neurons with delayed chemical synapses", Phys. Rev. A, vol. 78, no. 3, pp. 036211, 2008.

[42] R. F. Fox, "Stochastic versions of the Hodgkin-Huxley equations", Biophys. J., vol. 72, no. 5, pp. 2068-2074, 1997. 
